\title{
Effects of autophagy regulation of tumor-associated macrophages on radiosensitivity of colorectal cancer cells
}

\author{
LE-NING SHAO ${ }^{1 *}$, BAO-SONG ZHU ${ }^{1 *}$, CHUN-GEN XING ${ }^{1}$, \\ XIAO-DONG YANG ${ }^{1}$, WU YOUNG ${ }^{1}$ and JIAN-PING CAO ${ }^{2}$ \\ ${ }^{1}$ Department of General Surgery, The Second Affiliated Hospital of Soochow University, Suzhou, Jiangsu 215004; \\ ${ }^{2}$ Jiangsu Provincial Key Laboratory of Radiation Medicine and Protection, \\ Soochow University, Suzhou, Jiangsu 215123, P.R. China
}

Received May 11, 2015; Accepted January 4, 2016

DOI: $10.3892 / \mathrm{mmr} .2016 .4820$

\begin{abstract}
Tumor-associated macrophages (TAMs), a major component of the tumor microenvironment, are crucial to the processes of tumor growth, infiltration and metastasis, and contribute to drug resistance. The importance of TAMs in radiation resistance of colorectal cancer remains unclear. To investigate the effects of autophagy regulation of TAMs on the radiosensitivity of colorectal cancer cells, the current study induced TAM formation from THP-1 monocyte cells. Sequential treatment of THP-1 cells with PMA for $72 \mathrm{~h}$ and human recombinant interleukin- 4 for $24 \mathrm{~h}$ was used to stimulate THP-1 differentiation to TAMs. Expression of the cell surface markers CD68, CD204 and CD206, and changes to cell morphology were used to confirm successful differentiation. The TAMs were stimulated to promote or inhibit autophagy during co-culture with LoVo colorectal adenocarcinoma cells. The cells were irradiated, with subsequent measurement of LoVo colony formation and apoptosis. Additionally, the expression of p53, Bcl-2, survivin and Smac proteins was assessed by western blotting. Monodansylcadaverin staining was used to analyze the presence of autophagic vacuoles in TAM, and western blot analysis was used to assess the expression of Beclin-1, LC3B I and II, ATG-3,-5 and -7. The results demonstrated TAM autophagy to be markedly altered by rapamycin and bafilomycin A1 treatment. Following co-culture with TAMs, the colony formation rate and survival fraction of LoVo cells were significantly higher than those in the control group $(\mathrm{P}<0.05)$. It was further demonstrated that the regulation of autophagy in TAMs was able to inhibit the colony formation of
\end{abstract}

Correspondence to: Professor Chun-Gen Xing, Department of General Surgery, The Second Affiliated Hospital of Soochow University, 1055 Sanxian Road, Suzhou, Jiangsu 215004, P.R. China E-mail: xingcg@126.com

*Contributed equally

Key words: tumor-associated macrophages, autophagy, colorectal cancer, radiosensitivity
LoVo colorectal cancer cells. Upregulation of TAM autophagy using rapamycin exhibited more effective inhibition of LoVo colony formation than autophagy downregulation. Notably, apoptosis was significantly increased in LoVo cells when co-cultured with TAMs only, or with rapamycin-mediated autophagy upregulated TAMs, compared with LoVo cells cultured alone $(\mathrm{P}<0.01)$. Expression of $\mathrm{Bcl}-2$, survivin and $\mathrm{p} 53$ were reduced in LoVo cells co-cultured with TAMs, compared with the control group $(\mathrm{P}<0.05)$, whereas Smac expression was increased in the co-culture groups $(\mathrm{P}<0.01)$. It was demonstrated that rapamycin-mediated autophagy stimulation in TAMs led to reduced expression levels of survivin and Bcl-2, however, Smac expression was increased. The upregulation of autophagy in TAMs inhibited proliferation and induced apoptosis in colon cancer cells, and altered the expression of radiosensitivity-associated proteins. This data indicated that the radiosensitivity of colorectal cancer cells is associated with autophagy of TAM, and that stimulating TAM autophagy may increase the radiosensitivity of colorectal cancer cells.

\section{Introduction}

Colorectal cancer is one of the three leading causes of cancer-associated mortality worldwide, with $\sim 1,200,000$ new cases and 600,000 deaths annually (1). In China, the incidence and mortality rates of colorectal cancer have increased rapidly in the past several decades (2), with mortality generally resulting from tumor recurrence or metastasis (3). Metastasis to regional lymph nodes is critical in colorectal cancer tumor progression, affects prognosis, and occurs commonly in early-stage metastasis (4). Additionally, lymph node involvement often promotes further hematogenous metastasis (5). Following surgery, the 5-year survival rate of patients with early-stage colorectalcancer is $>90 \%$, however, the 5-year survival rate of advanced-stage patients is $<10 \%$ (6). Although several genes associated with lymph node metastasis have been reported, the molecular mechanisms of early-stage metastasis in colorectal cancer remain unclear (7-9). Thus, the identification of bio-markers associated with lymph node metastasis of colorectal cancer will benefit clinical evaluation and future treatment strategies.

Currently, the typical treatment for colorectal cancer is surgery combined with radiotherapy (10). Radiotherapy 
is important for the control of colorectal cancer lymph node metastasis and the prevention of local recurrence. Tumor-associated macrophages (TAMs) constitute 30-50\% of tumor stromal cells, and secrete a variety of cytokines to promote tumor growth and progression, including basic fibroblast growth factor, transforming growth factor- $\beta$ (TGF- $\beta$ ), platelet-derived growth factor (PDGF) and epidermal growth factor (11). The quantity of TAMs in tumor tissue is considered to be directly associated with poor disease prognosis (12). Previous studies in lymphoma and breast cancer have demonstrated that the growth of tumor cells in vitro was increased by co-culture with TAMs $(13,14)$. Therefore, to explore the interactions between TAMs and tumor cells, and elucidate the mechanism by which TAMs promote growth, it is important to develop novel processes and targets for the treatment of cancer.

Tumor cell autophagy has been investigated in numerous studies, however, there are few reports analyzing the regulation of autophagy in tumor stromal cells and how this impacts the biological characteristics of tumor cells. Therefore, the current study used co-culture of TAMs with colorectal cancer cells to investigate how autophagy of TAMs may influence the radiosensitivity of colon cancer.

\section{Materials and methods}

Cell culture and reagents. The following cell lines were used: LoVo low differentiated colon adenocarcinoma cell line (American Type Culture Collection, Manassas, VA, USA); and THP-1 human monocytic leukemia cell line (Shanghai Institute of Biochemistry and Cell Biology, Chinese Academy of Sciences, Shanghai, China). The cell lines were routinely cultured in RPMI-1640 medium (Gibco; Thermo Fisher Scientific, Inc., Waltham, MA, USA) supplemented with $10 \%$ fetal bovine serum (FBS; Gibco; Thermo Fisher Scientific, Inc.) and maintained at $37^{\circ} \mathrm{C}$ in a humidified environment with $5 \% \mathrm{CO}_{2}$. Rapamycin, bafilomycin $\mathrm{A} 1$ and phorbol-12-myristate-13-acetate (PMA) were purchased from Abcam (Cambridge, UK). Monoclonal mouse anti-human epitope 206 CD206 (cat. no. sc-58986), monoclonal mouse anti-human B-cell lymphoma-2 (Bcl-2; cat. no. sc-7382), polyclonal rabbit anti-human survivin (cat. no. sc-10811)and monoclonal mouse anti-human second mitochondria-derived activator of caspase (Smac; cat. no. sc-393118) antibodies were purchased from Santa Cruz Biotechnology, Inc. (Dallas, Texas, USA). Monoclonal mouse anti-human CD68 antibody (cat. no. sc-20060; Santa Cruz Biotechnology, Inc.), monoclonal mouse anti-human p53 (cat. no. LS-C43831; LifeSpan BioSciences, Inc., Seattle, WA, USA), polyclonal rabbit anti-human LC3B I and II (cat. no. L8918; Sigma-Aldrich) and mouse anti-human SR-AI/MSR/CD204 (cat. no. 351615; Novus Biologicals, Littleton, CO, USA) antibodies were also used. Monodansylcadaverine (MDC) was purchased from Sigma-Aldrich (St. Louis, MO, USA). An Autophagy Antibody Sampler kit (cat. no. 4445), including the ATG-3, ATG-5, ATG-7 and Beclin-1 antibodies, was purchased from Cell Signaling Technology, Inc. (Danvers, MA, USA) and the human recombinant interleukin-4 (IL-4; cat. no. 200-04) was obtained from Peprotech Inc. (Rocky Hill, NJ, USA). The Annexin V/FITC Apoptosis Detection kit was purchased from Roche Applied Science (Madison, WI, USA) and a Transwell chamber was purchased from Corning Incorporated (Corning, NY, USA).

Cell irradiation conditions. Cells were irradiated using a Primus linear accelerator (Siemens Medical Solutions USA, Inc., Malvern, PA, USA), 6MV X-ray vertical irradiation at $198 \mathrm{cGy} / \mathrm{min}$ and $25^{\circ} \mathrm{C}$ with a source skin distance of $100 \mathrm{~cm}$.

Stimulation and identification of TAMs. THP-1 cells $\left(4 \times 10^{6}\right)$ were cultured in medium containing $30 \mathrm{nM}$ PMA for $72 \mathrm{~h}$. Subsequently, $60 \mathrm{ng}$ IL-4 was added to the medium for a further $24 \mathrm{~h}$. Cells were collected and immunolabeled with antibodies against CD204, CD206 (1:1,000) and CD68 $(1: 1,000)$ to detect TAM markers. Cells were then stained with FITC-labeled goat anti-mouse IgG (H+L; cat. no. A0568; Beyotime Institute of Biotechnology, Shanghai, China) secondary antibody. Following staining, cells were washed with RPMI-1640 medium (Gibco; Thermo Fisher Scientific, Inc.) supplemented with 5\% fetal bovine serum (FBS; Gibco; Thermo Fisher Scientific, Inc.) twice and immediately analyzed by flow cytometry (Cytomic FC 500; Beckman Coulter, Inc., Brea, CA, USA). THP-1 cells treated with phosphate-buffered saline (PBS) for $72 \mathrm{~h}$, or with only PMA for $72 \mathrm{~h}$ were used as the control groups.

Autophagy of TAM analysis. All groups consisted of TAMs cultured in RPMI 1640 supplemented with PBS and treated as follows: i) The control group, 5\% FBS only; ii) the PBS group, $\mathrm{PBS}+10 \% \mathrm{FBS}$; iii) the autophagy upregulation group, $200 \mathrm{nM} / 1$ rapamycin for $48 \mathrm{~h}+10 \% \mathrm{FBS}$; and iv) the autophagy downregulation group, $50 \mathrm{nM} / 1$ bafilomycin A1 for $48 \mathrm{~h}+10 \% \mathrm{FBS}$. The fluorescent dye MDC (100 nM) was added to all groups following 2 PBS washes, then incubated for $30 \mathrm{~min}$ at $25^{\circ} \mathrm{C}$. The green fluorescence was detected by laser scanning confocal microscopy (micropublisher 3.3RTV; Olympus, Tokyo, Japan). Western blot was used to assess the expression of microtubule associated protein 1 light chain $3 \beta$-I and -II (LC3B I and II), Beclin-1 and autophagy related-3, -5 and -7 (ATG-3, -5 and -7). Proteins were extracted using a Total Protein Extraction kit (EMD Millipore, Billerica, MA, USA) and the protein concentration of lysates was determined using the bicinchoninic acid method (BCA Protein Quantification kit; Beyotime Institute of Biotechnology). The following antibodies were used to develop immunoreactive signals: LC3B I and II $(1: 1,000$; Sigma-Aldrich), polyclonal rabbit anti-human ATG-3 (cat. no. 3415; Cell Signaling Technology, Inc.), monoclonal rabbit anti-human ATG-5 (cat. no. 12994; Cell Signaling Technology, Inc.), monoclonal rabbit anti-human ATG-7 (cat. no. 8558; Cell Signalling Technology, Inc.), monoclonal rabbit anti-human Beclin-1 (cat. no. 3495; 1:1,000; Cell Signaling Technology, Inc.), goat polyclonal GAPDH (cat. no. sc-48167; Santa Cruz Biotechnology, Inc.) and rabbit polyclonal $\beta$-actin (cat. no. sc-7210; 1:1,000; Santa Cruz Biotechnology, Inc.). Densitometry was performed using AlphaImager 2200 system (Alpha Innotech, Santa Clara, CA, USA).

TAMs co-cultured with LoVo cells and radiation process. The non-contact co-culture group settings were as follows: i) LoVo cells alone, no radiation; ii) LoVo cells with 6 Gy irradiation; 
Table I. Percentage of monodansylcadaverine-activated TAMs.

Group

Fluorescent cells (\%)

TAM (5\% FBS)
TAM+PBS (10\% FBS)
TAM+rapamycin (10\% FBS)
TAM+bafilomycin A1 (10\% FBS)

$3.6 \pm 0.64$

$38.3 \pm 1.07$

$76.4 \pm 1.14$

$21.3 \pm 1.21$

TAM, tumor-associated macrophages; FBS, fetal bovine serum; PBS, phosphate-buffered saline.

iii) LoVo and TAMs; iv) the autophagy-upregulation group, LoVo and TAMs with rapamycin treatment; and v) the autophagy-downregulation group, LoVo and TAMs with bafilomycin A1 treatment. All groups containing TAMs were also exposed to $6 \mathrm{~Gy}$ irradiation. LoVo cells were cultured in the lower chamber, and TAMs in the upper chamber of a Transwell insert separated by a polycarbonate membrane, thus, preventing direct cell-cell contact, but permitting the exchange of soluble factors. LoVo cells $\left(4 \times 10^{5}\right)$ were seeded in the lower chamber of a 6 -well plate. TAMs $\left(8 \times 10^{5}\right)$ were seeded on the micropore membrane $(0.4 \mu \mathrm{m}$ diameter $)$ in the upper chamber of the Transwell unit. The cells were co-cultured at $37^{\circ} \mathrm{C}$ in a $5 \% \mathrm{CO}_{2}$ environment for $48 \mathrm{~h}$. Excluding the control group, all cells received a dose of $6 \mathrm{~Gy}$ irradiation and were then cultured for another $36 \mathrm{~h}$. LoVo cells were cultured for 2 weeks prior to colony counting. LoVo cells from the lower chambers were removed and seeded into 6-aperture plates at a density of 200 cells/well. Colony formation was observed in each group and the colonies were counted ( $\geq 50$ cells were considered a standard cloning). Colony formation rate $(\%)=$ cloning efficiency/seeded cells x 100. Cell survival fraction = cloning efficiency of the experimental group/control group cloning efficiency (each containing 10 samples in parallel).

Detection of apoptosis in LoVo cells by annexin $V /$ propidium iodide (PI) staining. The non-contact co-culture groups were as follows: i) LoVo cells alone; ii) LoVo and TAMs; iv) the autophagy-upregulation group, LoVo and TAMs with rapamycin treatment; and iv) the autophagy-downregulation group, LoVo and TAMs with bafilomycin A1 treatment. LoVo cells $\left(4 \times 10^{5}\right)$ were seeded in the lower chamber of the Transwell insert in 6 -well plates. TAMs $\left(8 \times 10^{5}\right)$ were seeded on the micropore membrane $(0.4 \mu \mathrm{m}$ diameter $)$ in the upper chamber. Cells were co-cultured at $37^{\circ} \mathrm{C}$ and $5 \% \mathrm{CO}_{2}$ environment for $48 \mathrm{~h}$. All 4 groups received a dose of $6 \mathrm{~Gy}$ irradiation and were cultured for a further $24 \mathrm{~h}$. LoVo cells in the lower chamber were collected, washed with cold PBS buffer twice, and centrifuged at $370 \mathrm{x}$ g for $5 \mathrm{~min}$. Cells were resuspended in $195 \mu \mathrm{l}$ annexin $\mathrm{V}$-fluorescein isothiocyanate (FITC) binding buffer, then $5 \mu \mathrm{l}$ annexin V-FITC and $10 \mu \mathrm{l}$ PI were added to the solution and incubated for $15 \mathrm{~min}$ in darkness. The excitation wavelength used for flow cytometry detection was $488 \mathrm{~nm}$, FITC was detected by a $515 \mathrm{~nm}$ wavelength bandpass filter and PI by a $560 \mathrm{~nm}$ filter (Cytomic FC 500; Beckman Coulter, Inc.).
Western blot analysis of Smac, survivin, Bcl-2 and p53 expression levels. Proteins were extracted using the Total Protein Extraction kit and the protein concentrations of lysates were determined using the BCA method as described above. Subsequently, proteins were separated by $10 \%$ SDS-PAGE. At the beginning of electrophoresis, the voltage was $80 \mathrm{~V}$, then after $\sim 40 \mathrm{~min}$, the voltage was increased to $120 \mathrm{~V}$ for $1.5-2 \mathrm{~h}$. Proteins were then transferred onto PVDF membranes. When the protein transfer was completed, the gel was stained with Coomassie Brilliant Blue. PVDF membranes were washed with $10 \mathrm{X}$ TBS for $10 \mathrm{~min}$ at room temperature and then immersed with blocking buffer $[100 \mathrm{ml}$ Tris-buffered saline with Tween 20 (TBST) and $5 \mathrm{~g}$ skim milk powder (MeiJi Dairies Corporation, Tokyo, Japan)] for $1 \mathrm{~h}$. The membranes were incubated with the following primary antibodies: Mouse anti-human Smac (1:1,000; Santa Cruz Biotechnology, Inc.), rabbit anti-human survivin (1:1,000; Santa Cruz Biotechnology, Inc.), mouse anti-human Bcl-2 (1:1,000; Santa Cruz Biotechnology, Inc.), mouse anti-human p53 (1:1,000; LifeSpan BioSciences, Inc., Seattle, WA, USA) and $\beta$-actin $(1: 1,000)$ for $1 \mathrm{~h}$ at room temperature to develop immunoreactive signals. The membranes were then washed with 10X TBS for $30 \mathrm{~min}$ at room temperature and incubated with HRP-labeled goat anti-rabbit IgG (cat. no. A0208; Beyotime Institute of Biotechnology) and goat anti-mouse IgG (cat. no. A0216; Beyotime Institute of Biotechnology) secondary antibodies. Densitometry was performed using AlphaImager 2200 system and Quantity software.

Statistical analysis. All experiments were performed a minimum of 4 times. All results are expressed as the mean \pm standard deviation. Differences among multiple groups were analyzed by one-way analysis of variance with a Dunnett's multiple comparison post-hoc test. $\mathrm{P}<0.05$ was considered to indicate a statistically significant difference. Statistical analyses were performed using GraphPad Prism 6.0 (GraphPad Software, Inc., La Jolla, CA, USA).

\section{Results}

Induction of THP-1 differentiation and the identification of TAMs. THP-1 human leukemia cells were treated with PMA for $72 \mathrm{~h}$ and subsequently incubated with human recombinant IL-4 for $24 \mathrm{~h}$. The cell morphology was observed (Fig. 1A) and the expression of the TAM cell surface markers, CD68, CD204 and CD206 were detected by flow cytometry (Fig. 1B). The combined effects of PMA and IL-4 led to the differentiation of THP-1 cells into TAMs. The cells lost their spherical morphology, appeared more irregular, and became adherent. Cells stimulated with PMA and IL-4 exhibited increased expression of CD68, CD204 and CD206 (67.32 \pm 1.58 , $33.49 \pm 2.11$ and $45.42 \pm 2.65 \%)$ compared with control THP-1 cells $(39.69 \pm 1.59,8.56 \pm 1.25$ and $7.93 \pm 0.53 \%$ ) (Fig. 1B; $\mathrm{P}<0.01)$. The differentiated TAM group demonstrated the highest expression levels of CD68, CD204 and CD206 cell surface markers among the 3 groups, consistent with previous studies (15-17) (Fig. 1C).

TAM autophagy analysis. MDC-positive cells were almost undetectable in the control group $(3.6 \pm 0.64 \%$; Fig. 2Aa; 
A
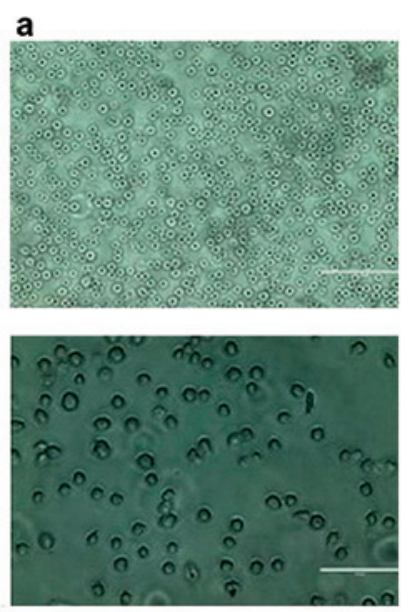

b
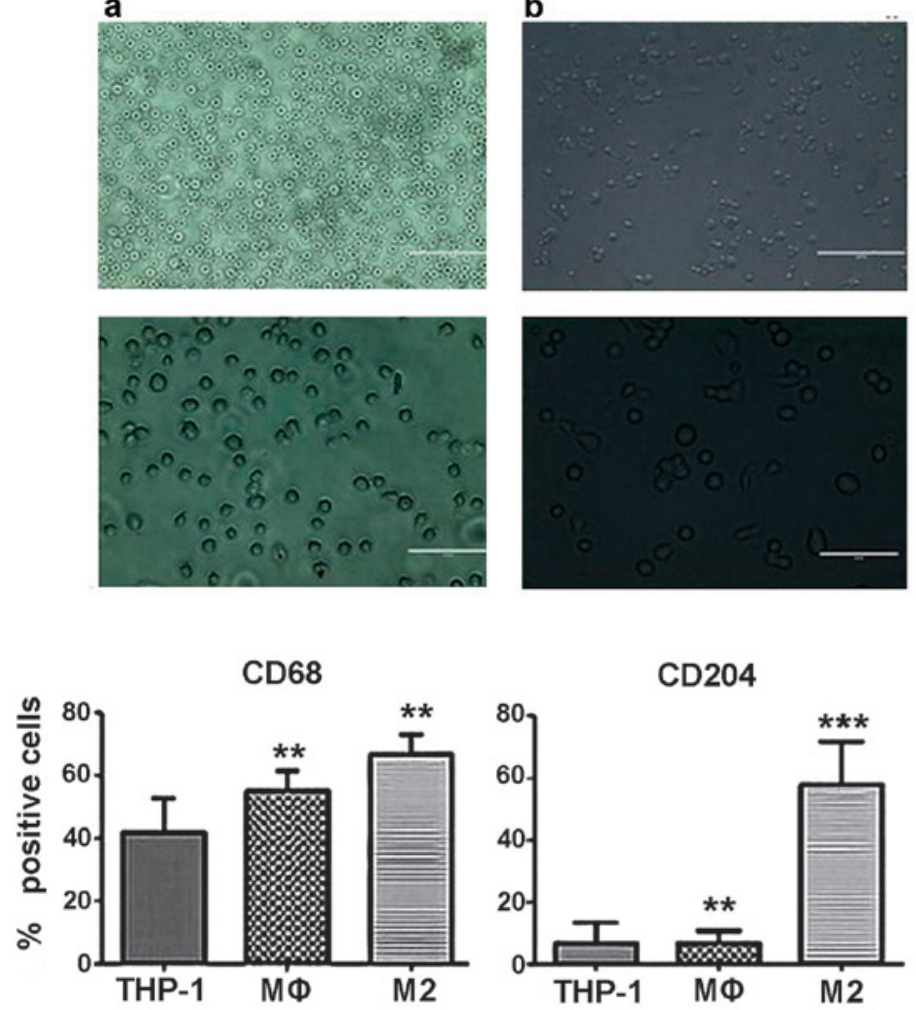

CD204
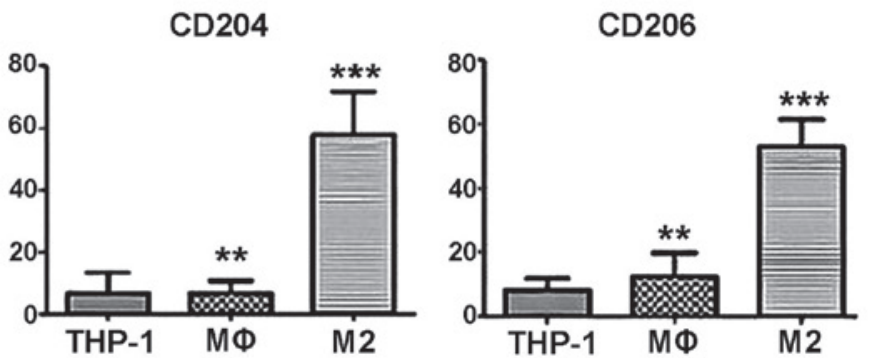
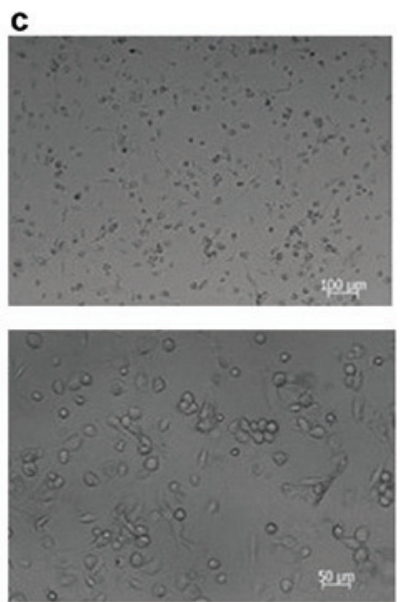

B

$\mathbf{C}$

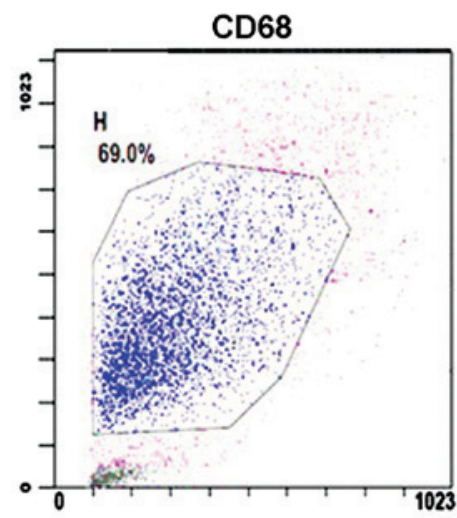

\section{Surface CD molecules of TAM}

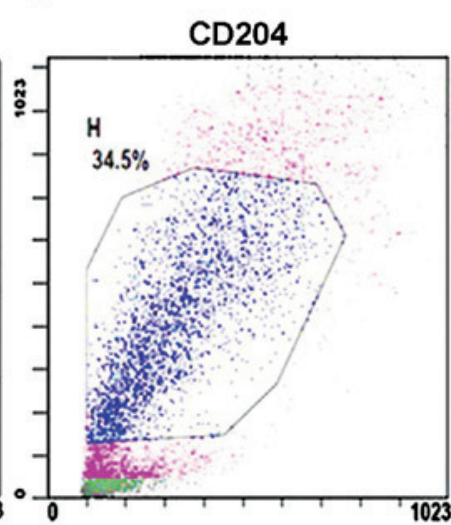

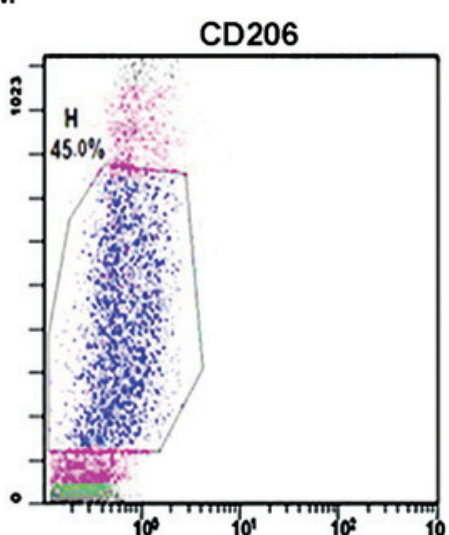

Figure 1. Induction of human monocytic leukemia THP-1 cells to TAMs. (Aa) Phosphate-buffered saline treatment; (Ab) PMA treatment ( $30 \mathrm{nM}$ ) for $72 \mathrm{~h}$ (M0); or (Ac) PMA treatment + $60 \mathrm{ng}$ IL-4 for another $24 \mathrm{~h}$ (M2). Top panel, low magnification (scale bar=100 $\mu \mathrm{M}$ ); lower panel, high magnification (scale bar=50 $\mu \mathrm{M}$ ). (B) Flow cytometry detection of surface CD molecules as markers of THP-1, M0 and M2 cells, with THP-1 cells as the control group. CD68, CD204 and CD206 expression were $39.69 \pm 1.59,8.56 \pm 1.25$ and $7.93 \pm 0.53 \%$ in THP-1 cells, $49.72 \pm 1.82,7.90 \pm 1.19$ and $14.39 \pm 0.85 \%$ in M0 cells and $67.32 \pm 1.58$, $33.49 \pm 2.11$ and $52.42 \pm 2.65 \%$ in TAMs. ${ }^{* *} \mathrm{P}<0.01$ and ${ }^{* * *} \mathrm{P}<0.001$ vs. the THP-1 group. (C) Surface CD molecule expression in TAMs. Processed flow cytometry detection was performed four times, and representative results are presented. TAM, tumor-associated macrophages; $\mathrm{CD}$, cluster of differentiation.

Table I) and in the untreated group, the rate of MDC-positive cells was $38.3 \pm 1.07 \%$ (Fig. 2Ab; Table I). By contrast, following 48-h treatment with rapamycin or bafilomycin A1 for $48 \mathrm{~h}$, the rate of MDC-positive cells in the autophagy-upregulation group was $76.4 \pm 1.14 \%$ (Fig. 2Ac; Table I) and in the autophagy-downregulation group it was $21.3 \pm 1.21 \%$ (Fig. 2Ad; Table I). Western blot analysis was performed to detect the expression of LC3B I and II in the 4 groups, and the highest relative expression level of the LC3Bs was observed in the autophagy-upregulation group, in which a significant increase was observed compared with the control group $(\mathrm{P}<0.001$; Fig. 2B). Expression levels of ATGs and Beclin-1 were highest in the autophagy-upregulation group, demonstrating that rapamycin treatment successfully stimulated autophagy of TAMs (Fig. 2C). The relative expression level of ATGs and Beclin-1 in the autophagy-downregulation group was the lowest of the 3 co-culture groups, demonstrating that bafilomycin A1 suppressed autophagy (Fig. 2C). Although MDC-positive cells were almost undetectable in the control group, western blot detected ATG-3, -5 and -7 and Beclin- 1 expression $(0.74 \pm 0.01$, $2.38 \pm 0.02,2.43 \pm 0.03$ and $1.01 \pm 0.03$; Fig. $2 \mathrm{Ca}$ ). This may be due to the cells being cultured in a closed environment, with changes of $\mathrm{pH}$ and nutrient consumption spontaneously stimulating autophagy, resulting in detection at the protein level. 
A
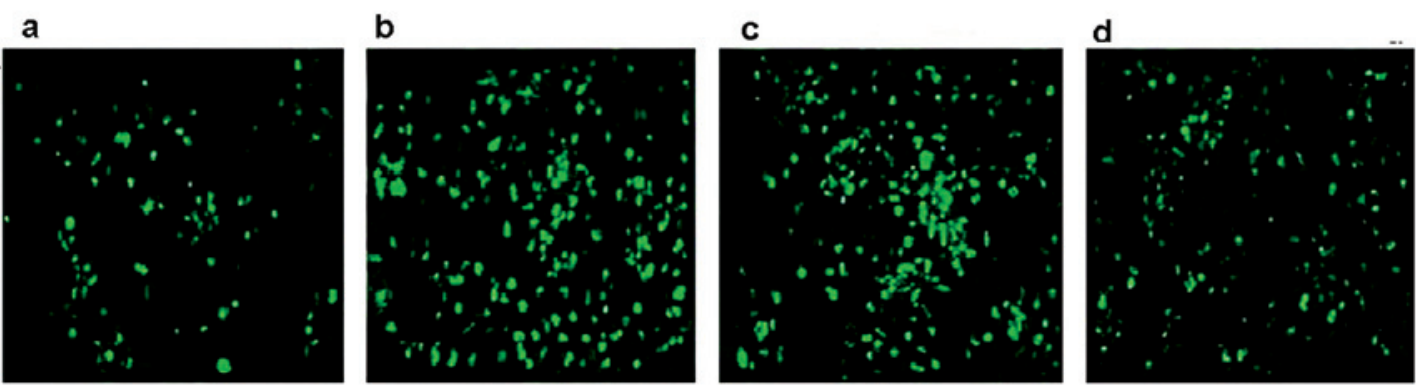

B
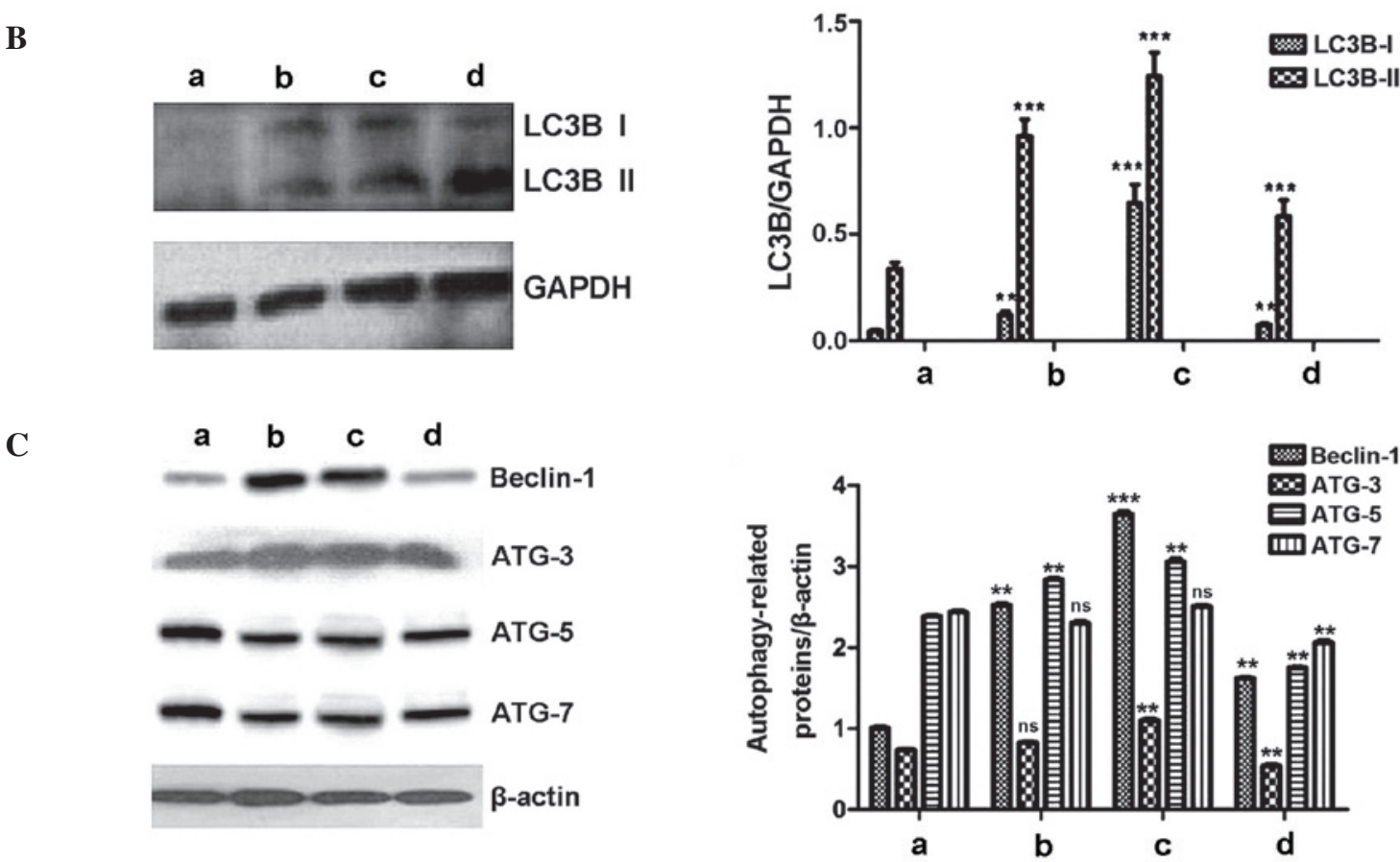

Figure 2. TAM autophagy analysis. The treatment groups were as follows: a, $5 \%$ FBS; b, PBS + 10\% FBS; c, rapamycin + 10\% FBS; and d, bafilomycin A1 + 10\% FBS. (A) MDC-positive autophagy capsules in TAMs. MDC is absorbed by autophagosomes and accumulates in the cytoplasm of TAMs, the green fluorescence represents MDC-positive autophagy capsules in TAMs. The frequency of green cells indicates degree of autophagy. (B) Western blot analysis detected the expression of LC3B I and II. (C) Western blot analysis indicated the expression levels of Beclin-1 and ATG-3, -5 and -7 in the different TAM treatment groups. ${ }^{* *} \mathrm{P}<0.01$ and ${ }^{* * * *} \mathrm{P}<0.001$ vs. TAMs cultured in RPMI-1640 medium supplemented with 5\% FBS. LC3B, microtubule associated protein 1 light chain $3 \beta$; TAM, tumor-associated macrophage; MDC, monodansylcadaverine; PBS, phosphate-buffered saline; ATG, autophagy related; ns, not significant.

Colony formation and western blot analysis of Smac, survivin, Bcl-2 and p53 expression in LoVo cells. The colony formation rate of LoVo cells cultured alone, without irradiation, was $10.01 \pm 0.48 \%$, and the cell survival fraction was $100 \%$ (Fig. 3A and B; Table II). When LoVo cells received 6 Gy irradiation, the colony number was reduced significantly compared with the control group $(\mathrm{P}<0.01)$, the colony formation rate was $3.98 \pm 0.73 \%$ and the cell survival fraction was $39.7 \pm 1.14 \%$ (Fig. $3 \mathrm{Ab}$ and $\mathrm{B}$; Table II). When LoVo cells were co-cultured with TAMs and received 6 Gy irradiation, the colony number increased significantly compared with the LoVo cells alone with 6 Gy radiation, and the colony formation rate was increased to $7.12 \pm 0.14 \%$ (Fig. 3Ac and B; Table II). This demonstrated that TAMs promote the proliferation of LoVo cells and reduce the cell death caused by radiation. When the autophagy status of TAM was altered by treatment with rapamycin or bafilomycin A1, the colony formation rate of the LoVo cells was affected. In the autophagy-upregulation group, the colony formation rate was $4.21 \pm 0.18 \%$, which was reduced compared with the downregulation group $(5.28 \pm 0.23 \%)$ and untreated group $(7.12 \pm 0.14 \%)$ (Fig. 3B; Table II). Western blot analysis was performed to measure the expression levels of Bcl-2, Smac, survivin and p53. Relative expression of Bcl-2 in the untreated TAM group was $0.24 \pm 0.02$, in the autophagy-upregulation group it was $0.08 \pm 0.01$, whilst in the autophagy-downregulation group it was $0.42 \pm 0.02$ (Fig. 3C). In the control group of LoVo cells cultured alone, the relative Bcl-2 expression level was $0.61 \pm 0.05$. Relative expression levels of Smac in the TAM co-culture untreated, autophagy-upregulated and -downregulated groups were $1.26 \pm 0.03,1.49 \pm 0.24$ and $0.85 \pm 0.03$, respectively, and $0.68 \pm 0.03$ in the control group (Fig. 3C). Expression levels of survivin in the TAM co-culture untreated, autophagy-upregulated and -downregulated groups were $0.48 \pm 0.01,0.23 \pm 0.02$ and $0.55 \pm 0.05$, respectively, and $0.80 \pm 0.05$ in the control group. Expression levels of p53 in the TAM co-culture untreated, autophagy-upregulated and -downregulated groups were $0.14 \pm 0.006,0.10 \pm 0.008$ and $0.07 \pm 0.012$, respectively, and $0.12 \pm 0.011$ in the control group (Fig. 3C). 
Table II. Colony formation rate and survival fraction of LoVo cells.

\begin{tabular}{lrr}
\hline Group & Colony formation rate (\%) & Survival fraction $(\%)$ \\
\hline LoVo (6 Gy irradiation) & $3.98 \pm 0.73$ & $39.70 \pm 1.14$ \\
LoVo+TAM (PBS + 6 Gy irradiation) & $7.12 \pm 0.14$ & $71.12 \pm 0.34$ \\
LoVo+TAM (rapamycin + 6 Gy irradiation) & $4.21 \pm 0.18$ & $42.05 \pm 0.27$ \\
LoVo+TAM (bafilomycin A1 + 6 Gy irradiation) & $5.28 \pm 0.23$ & $52.74 \pm 0.42$ \\
LoVo (without irradiation) & $10.01 \pm 0.48$ & 100
\end{tabular}

TAM, tumor-associated macrophages; PBS, phosphate-buffered saline.

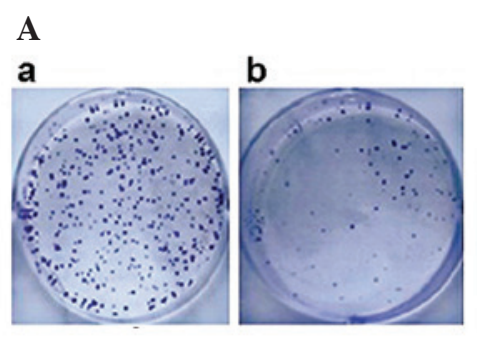

C

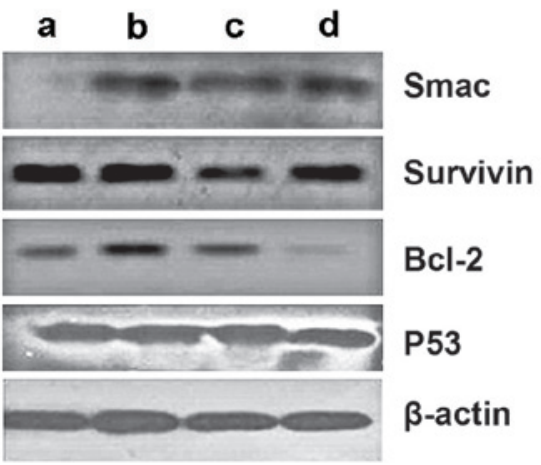

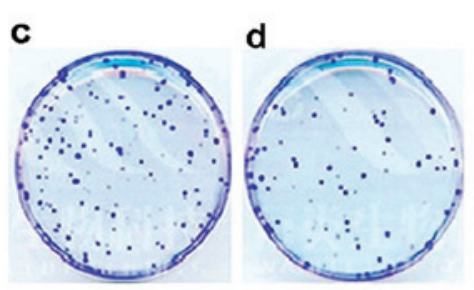

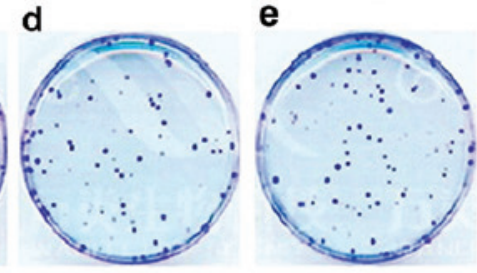

B

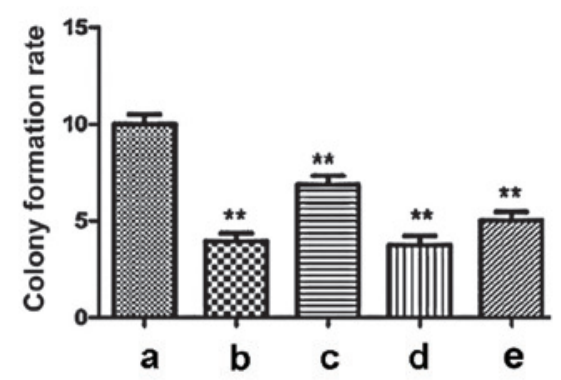

Figure 3. Colony formation and western blot analysis of the expression of Smac, survivin, Bcl-2 and p53 in LoVo cells. (A) Clonogenesis of LoVo cells. Treatment groups were as follows: a, LoVo cells + no radiation; b, LoVo cells + 6 Gy radiation; c, LoVo cells + TAM co-culture with 6 Gy radiation; d, LoVo + autophagy-upregulation TAM co-culture (rapamycin) with 6 Gy radiation; and e, LoVo + autophagy-downregulation TAM co-culture (bafilomycin A1) with 6 Gy radiation. (B) Colony formation rate of LoVo cells in the 5 groups. ${ }^{* *} \mathrm{P}<0.01$ and ${ }^{* * *} \mathrm{P}<0.001$ vs. LoVo cells cultured alone without irradiation. (C) Expression of Bcl-2, Smac, survivin and p53. Treatment groups were as follows: a, LoVo cells alone; b, LoVo cells + untreated TAM co-culture; c, LoVo + autophagy-upregulated TAM co-culture (rapamycin); and d, LoVo + autophagy-downregulated TAM co-culture (bafilomycin A1). ${ }^{*} \mathrm{P}<0.05,{ }^{* *} \mathrm{P}<0.01$ and ${ }^{* * *} \mathrm{P}<0.001$ vs. LoVo cells cultured alone without irradiation. TAM, tumor-associated macrophage; Smac, second mitochondria-derived activator of caspase.

When LoVo cells were co-cultured with TAMs, their colony-forming ability was improved and resistance to radiotherapy was enhanced. The results of the current study indicate that the autophagy status of TAMs may influence the proliferation and radiosensitivity-associated protein expression in LoVo cells. Therefore, the upregulation of autophagy in TAMs may reduce colony formation and enhance the anticancer effects of radiotherapy on colorectal cancer cells.

Annexin VIPI double staining flow cytometry as detection of apoptosis. Apoptosis of LoVo cells was measured by annexin V/PI double staining and flow cytometry. The percentages of apoptotic cells in the untreated, autophagy-upregulation and -downregulation groups were $37.49 \pm 3.42,45.73 \pm 3.49$ and $27.79 \pm 2.59 \%$, respectively. The percentage of apoptotic cells detected when LoVo cells were cultured alone was $25.19 \pm 3.46 \%$ (Fig. 4A; Table III). The highest degree of apoptosis occurred in the autophagy-upregulated TAM group (Fig. 4A; Table III). Therefore, the upregulation of TAM autophagy promoted the apoptosis of LoVo cells. The proportion of dead cells in the control group, including necrotic and apoptotic cells (62.13\%), was higher than that in the autophagy-downregulation group $(47.44 \%)$ as presented in Fig. 4A and Table III. The group with the highest number of necrotic cells was the control group, $36.94 \pm 0.48 \%$, compared with $19.65 \pm 0.96 \%$ in the autophagy-downregulation group. Therefore, when LoVo cells were co-cultured with TAMs, radiation-induced apoptosis was enhanced, and the upregulation of TAM autophagy significantly promoted the apoptosis of LoVo cells, improving the radiosensitivity of colorectal cancer cells. 
Table III. Proportion of apoptotic and necrotic LoVo cells.

\begin{tabular}{lcc}
\hline Group & Apoptotic cells (\%) & Necrotic cells (\%) \\
\hline LoVo (6 Gy irradiation) & $25.19 \pm 3.46$ & $36.94 \pm 0.48$ \\
LoVo+TAM (PBS + 6 Gy irradiation) & $37.49 \pm 3.42$ & $30.78 \pm 0.69$ \\
LoVo+TAM (rapamycin + 6 Gy irradiation) & $45.73 \pm 3.49$ & $34.12 \pm 0.89$ \\
LoVo+TAM (bafilomycin A1 + 6 Gy irradiation) & $27.79 \pm 2.59$ & $19.65 \pm 1.10$
\end{tabular}

TAM, tumor-associated macrophages; PBS, phosphate-buffered saline.

A

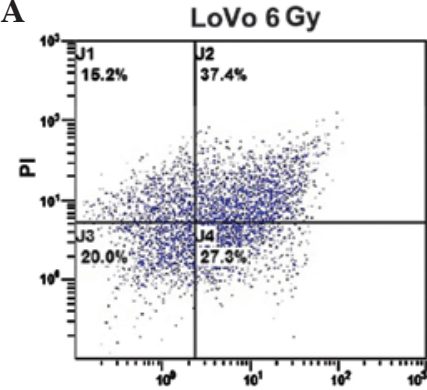

LoVo+TAM (Rapamycin) 6 Gy

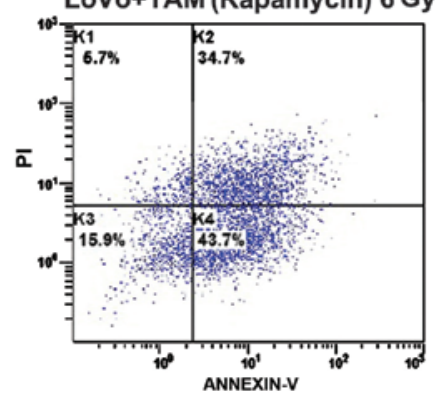

LoVo+TAM (PBS) 6 Gy

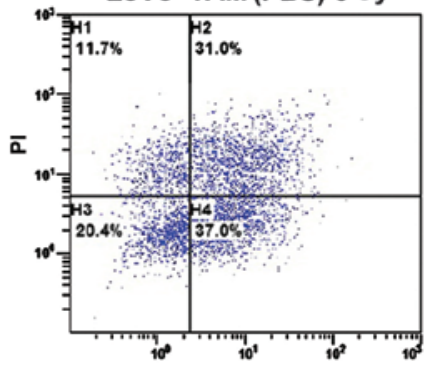

LoVo+TAM (Bafilomycin A1) 6 Gy

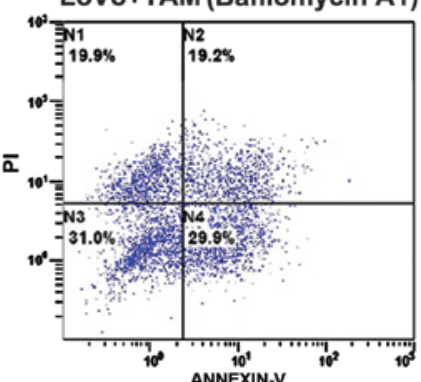

B

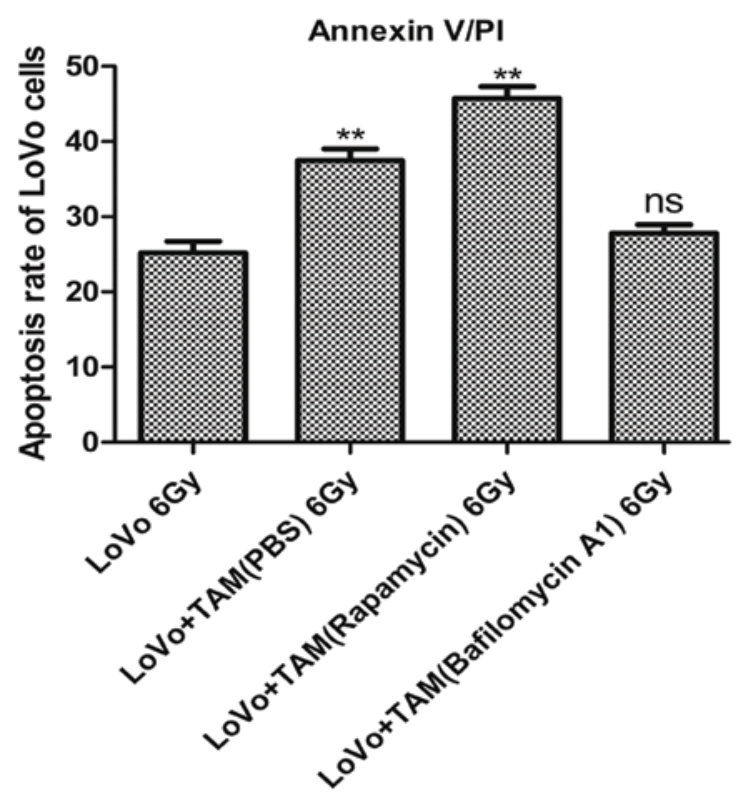

Figure 4. Apoptosis of LoVo detected by flow cytometry. (A) Upper right quadrant represents necrotic cells; lower right quadrant represents apoptotic cells; upper left quadrant represents disrupted cells as a result of the extraction method. (B) Apoptosis rate of LoVo cells in the 4 groups. ${ }^{* *} \mathrm{P}<0.01$ vs. the LoVo 6 Gy group. TAM, tumor-associated macrophage; PI, propidium iodide; ns, not significant; PBS, phosphate-buffered saline.

\section{Discussion}

It has long been recognized that cancer cells are not the only component of solid tumors. Tumor growth does not occur independently, it has a complicated and close association with tumor stromal cells and the extracellular matrix within the tumor microenvironment $(18,19)$. Stromal cells interact with tumors through a variety of signaling factors, contributing to tumor proliferation, invasion, infiltration and metastasis to distant sites (20).

TAMs are an integral part of the tumor microenvironment and are important in numerous processes, including tumor proliferation, invasion and metastasis $(21,22)$. TAMs in the tumor microenvironment have an M2 phenotype. Compared with normal macrophages, their antigen-presenting function and stimulation of type I adaptive immune responses are weak (21). Additionally, TAMs inhibit T cell proliferation and cytotoxic activity, induce tumor angiogenesis, promote malignant proliferation and aid tumor tissue repair $(22,23)$. Wyckoff et al (13) observed that greater TAM infiltration of tumor tissue was associated with worse disease prognosis in patients. Studies have also confirmed that TAMs secrete TGF- $\beta$, PDGF, tumor necrosis factor- $\alpha$, matrix metalloproteinase-9 and other factors, to promote tumor angiogenesis and accelerate tumor growth $(21,24)$. Previous studies have demonstrated that TAMs secrete PDGF, IL-1 and -6 to aid tumor cell survival and reduce tumor radiosensitivity $(15,16,25)$. Thus, the study of TAMs may uncover novel methods to improve the results of radiotherapy.

Autophagy is the degradation system of cells. By forming an autophagic-palade, cell fractions, including cellular protein and organelles, are passed to the lysosome for degradation, maintaining the metabolic balance of the cell (17). It has been observed that defects in autophagy can promote tumor development. When a tumor is progressing, cancer cells are under hypoxic stress and nutrient-limited conditions; this activates autophagy to degrade denatured proteins and damaged organelles, providing nutrients and energy to enable tumor cells to survive (26). The regulatory mechanisms of autophagy are complex. The mechanistic target of rapamycin (mTOR) kinase, is an important regulator of cell growth and development, and negatively regulates autophagy. The anti-fungal 
drug, rapamycin, can inhibit the activity of mTOR leading to autophagy activation (27). The current study used a rapamycin derivative, RAD001, to upregulate cellular autophagy. In the autophagy process, autophagic vesicles combine with lysosomes. The enzyme $\mathrm{H}^{+}$-ATPase is required for the acidification of vesicles, enabling mature lysosomal autophagy and autophagic degradation function. Bafilomycin A1 is a macrocyclic antibiotic that inhibits vesicle-type $\mathrm{H}^{+}$-ATPase, and therefore, acts as an inhibitor of autophagy (28).

In the current study, using previously described methods (29-31), PMA and IL-4 were used to induce human monocytic leukemia THP-1 cells to differentiate into TAMs. Differentiation was identified by observing changes to cell morphology and the expression of cell surface markers. Subsequently, rapamycin and bafilomycin A1 were used to stimulate and inhibit the autophagy of TAM.

MDC is a specific marker of autophagy that is absorbed by cells and accumulates in autophagic vesicles. The current study used fluorescence microscopy to observe MDC-positive autophagic vesicles. Autophagic vesicles have a punctate structure, and are located in the cytoplasm and nucleus. LC3 proteins are widely recognized as molecular markers of autophagy. LC3s are similar to the yeast proteins Apg8/Aut7/Atg8 and are important in the autophagy process. LC3 proteins are cleaved immediately by Atg4 at their carboxy-terminus following synthesis, producing LC3-I, which is localized in the cytoplasm. During the process of autophagy, LC3-I is modified by a ubiquitin-like system, involving the proteins ATG-3 and -7, resulting in a $14-\mathrm{kD}$ protein, LC3-II, which localizes to the autophagic body. Thus, the presence of autophagic bodies and the detection of LC3-I and LC3-II are treated as molecular markers of autophagy. There are three types of LC3 expressed in mammalian cells, including LC3A, B and C. They are required for the post-translational modifications associated with autophagy. However, only LC3B is expressed in autophagic structures, therefore, the expression of LC3B was used as a marker of autophagy in the current study. The number of MDC-stained autophagic vesicles (Fig. 2A) and the expression of LC3B (Fig. 2B) demonstrated that autophagy of TAMs was up- and downregulated by rapamycin and bafilomycin A1, respectively. The expression levels of ATGs and Beclin-1 were also altered following drug treatment (Fig. 2C).

Tumor radiosensitivity indicators include apoptosis, proliferation, cell survival fraction and expression of the tumor suppressor gene, p53 and its downstream targets. In the current study, apoptosis, proliferation, cell survival and the expression levels of p53, Smac, survivin and Bcl-2 were selected as an evaluation index of LoVo cell radiosensitivity.

Smac is a mitochondrial apoptotic regulatory protein. The N-terminal region of Smac associates with the BIR domains of survivin and inhibitor of apoptosis protein (IAP) family members, suppressing their anti-apoptotic activity. The inhibition of X-linked IAP (XIAP) is an important process. Smac and XIAP are important regulatory factors in the apoptotic pathway, which is closely associated with tumor development and radiation-induced apoptosis. During radiation-induced apoptosis, the BIR3 domain of XIAP binds with Smac, so it cannot associate with the initiation factor caspase 9 to stimulate the apoptotic cascade and induce apoptosis. Zheng et al (32) observed that the upregulation of Smac expression can enhance ionizing radiation-induced apoptosis and increase the sensitivity of HeLa cells to ionizing radiation. Giagkousiklidis et al (33) demonstrated that Smacdx molecule analogs can enhance radiation-induced apoptosis by antagonizing IAP and increasing caspase activity. These results suggest that therapeutically targeting Smac is a potential method to improve radiosensitivity in oncology. The IAP family is a group of anti-apoptotic proteins. The family have homologous sequences at the BIR and the RING finger domains, and can inhibit apoptosis and promote tumorigenesis. Smac is known to exist in mammalian cells and can directly inhibit IAP proteins. Studies have demonstrated that Smac expression is reduced in colon cancer cells $(32,34)$, suggesting that enhanced expression of Smac may improve the radiosensitivity of colorectal cancer.

Survivin is a member of the IAP family. It is not strongly expressed in differentiated mature tissue, however, survivin is highly expressed in embryonic tissue and stem cells. Analysis of fresh specimens identified that survivin is highly expressed in colon cancer, pancreatic cancer and other malignant tumors (35). Therefore, the inhibition of survivin expression may promote tumor cell apoptosis. Currently, studies have confirmed that reduced expression of survivin can induce apoptosis in various tumor cell lines in vitro, can slow tumor growth in experimental animal models and increase the sensitivity of tumors to various chemotherapeutic drugs and radiation (36). Rödel et al (37) detected the expression of survivin in several colon cancer cell lines (SW480, LoVo, SW48 and CT215), and observed that survivin was negatively correlated with spontaneous and radiation-induced apoptosis. Higher survivin expression levels were associated with decreased spontaneous apoptosis and improved resistance to radiation.

In the current study, the expression levels of Smac were increased, and survivin levels decreased in the TAM/LoVo co-culture groups compared with the LoVo alone control group $(\mathrm{P}<0.01)$. Downregulation of TAM autophagy increased survivin expression in LoVo cells and inhibited the expression of Smac compared with the control group $(\mathrm{P}<0.01)$. Upregulation of TAM autophagy inhibited survivin expression in LoVo cells and increased the expression of Smac compared with the LoVo cells alone. Following irradiation, apoptosis in the TAM autophagy-upregulation group was higher than all other groups. The results indicated that apoptosis is closely associated with the expression of Smac and survivin. Upregulation of TAM autophagy may inhibit survivin expression and increase Smac expression to promote the caspase cascade pathway, inducing apoptosis and increasing the radiosensitivity of LoVo cells.

The Bcl-2 family includes pro-apoptotic and anti-apoptotic proteins. Bcl-2, Bcl-xL and Bcl-w are anti-apoptotic family members. In the present study, western blot demonstrated that Bcl-2 expression in the autophagy-upregulation group was the lowest of all groups, whilst the rate of apoptosis was highest in this group. By contrast, the autophagy-downregulation group exhibited the highest $\mathrm{Bcl}-2$ expression and the lowest LoVo apoptosis rate of the 3 co-culture groups. The current study demonstrated that co-culture with TAMs increased the expression of p53 in LoVo cells, and that the expression of p53 was influenced by the autophagy status of TAMs ( $P<0.05$ vs. the control group). 
The cell survival fraction and colony formation of LoVo cells were also analyzed. Following irradiation, the colony formation rate in the untreated TAM co-culture group was increased compared with the LoVo cell only group, and its surviving fraction was the highest of all groups. Compared with when the colon cancer cells were cultured alone, the co-culture with TAM promoted the proliferation of LoVo cells, and proliferation was altered by the autophagy status of the TAMs. The colony formation rate of LoVo cells in the autophagy-upregulation group was lower than the untreated and autophagy-downregulation co-culture groups. When LoVo cells received 6 Gy irradiation, the survival fraction of LoVo cells was significantly decreased compared with LoVo cells cultured alone without irradiation. This indicates that the upregulation of TAM autophagy may suppress the effect of TAM on the promotion of tumor proliferation. Correlational studies have demonstrated that TAMs secrete IL-10, resulting in reduced activity of $\mathrm{NF}-\kappa \mathrm{B}$ and inhibition of transcriptional control of cell division (38). Therefore, the present study hypothesized that TAMs may act via the secretion of cytokines such as IL-10, to suppress NF- $\mathrm{B}$ activity, improving the efficiency of gene transcription to promote cell proliferation.

Drug intervention by rapamycin and bafilomycin Al was used to change the autophagy status of TAMs. This directly affected the biological behavior of TAMs, and altered the levels of proliferation, apoptosis and expression of radiosensitivity-associated proteins in tumor cells. Thus, the regulation of TAM autophagy changes the biological behavior of LoVo cells. Upregulation of TAM autophagy can inhibit the proliferation and promote apoptosis of colorectal cancer cells, thus, improving the efficacy of radiotherapy.

\section{Acknowledgements}

The present study was supported by grants from the National Natural Science Foundation of China (no. 81172348), the Jiangsu Provincial Key Laboratory of Radiation Medicine and Protection (no. KJS1334) and Suzhou Science and Education Guardian Youth Science and Technology Project (no. 2011010).

\section{References}

1. Jemal A, Bray F, Center MM, Ferlay J, Ward E and Forman D: Global cancer statistics. CA Cancer J Clin 61: 69-90, 2011.

2. Huang Z, Huang D, Ni S, Peng Z, Sheng W and Du X: Plasma microRNAs are promising novel biomarkers for early detection of colorectal cancer. Int J Cancer 127: 118-126, 2010.

3. Gutman M and Fidler IJ: Biology of human colon cancer metastasis. World J Surg 19: 226-234, 1995.

4. Sasaki H, Miura K, Horii A, Kaneko N, Fujibuchi W, Kiseleva L, Gu Z, Murata Y, Karasawa H, Mizoi T, et al: Orthotopic implantation mouse model and cDNA microarray analysis indicates several genes potentially involved in lymph node metastasis of colorectal cancer. Cancer Sci 99: 711-719, 2008.

5. Sleeman JP: The lymph node as a bridgehead in the metastatic dissemination of tumors. Cancer Res 157: 55-81, 2000.

6. Xu N, Qiu H and Ding Y: The relation between DNA replication error and clinicopathological features of colorectal carcinoma. Zhonghua Bing Li Xue Za Zhi 27: 359-361, 1998 (In Chinese).

7. Lin Y, Buckhaults PJ, Lee JR, Xiong H, Farrell C, Podolsky RH, Schade RR and Dynan WS: Association of the actin-binding protein transgelin with lymph node metastasis in human colorectal cancer. Neoplasia 11: 864-873, 2009.

8. Akagi T, Hijiya N, Inomata M, Shiraishi N, Moriyama M and Kitano S: Visinin-like protein-1 overexpression is an indicator of lymph node metastasis and poor prognosis in colorectal cancer patients. Int J Cancer 131: 1307-1317, 2012.
9. Toiyama Y, Yasuda H, Saigusa S, Tanaka K, Inoue Y, Goel A and Kusunoki M: Increased expression of Slug and Vimentin as novel predictive biomarkers for lymph node metastasis and poor prognosis in colorectal cancer. Carcinogenesis 34: 2548-2557, 2013.

10. Siegel R, DeSantis C, Virgo K, Stein K, Mariotto A, Smith T, Cooper D, Gansler T, Lerro C, Fedewa S, et al: Cancer treatment and survivorship statistics, 2012. CA Cancer J Clin 62: 220-241, 2012

11. Yue ZQ, Lui YP, Ruan JS, Zhou L and Lu Y: Tumor-associated macrophages: A novel potential target for cancer treatment. Chin Med J (Engl) 125: 3305-3311, 2012.

12. Rigo A, Gottardi M, Zamò A, Mauri P, Bonifacio M, Krampera M,Damiani E,Pizzolo G and Vinante F: Macrophages may promote cancer growth via a GM-CSF/HB-EGF paracrine loop that is enhanced by CXCL12. Mol Cancer 9: 273-279, 2010.

13. Wyckoff J, Wang W, Lin EY, Wang Y, Pixley F, Stanley ER, Graf T, Pollard JW, Segall J and Condeelis J: A paracine loop between tumor cells and macrophages is required for tumor cell migration in mammary tumors. Cancer Res 64: 7022-7029, 2004.

14. Canioni D, Salles G, Mounier N, Brousse N, Keuppens M, Morchhauser F, Lamy T, Sonet A, Rousselet MC, Foussard C, et al: High numbers of tumor-associated macrophages have an adverse prognostic value that can be circumvented by rituximab in patients with follicular lymphoma enrolled onto the GELA-COELAMS FL-2000 trial. J Clin Oncol 26: 440-446, 2008.

15. Kuwahara Y,Oikawa T, Ochiai Y, Roudkenar MH, Fukumoto M, Shimura T, Ohtake Y, Ohkubo Y, Mori S, Uchiyama Y, et al: Enhancement of autophagy is a potential modality for tumors refractory to radiotherapy. Cell Death Dis 2: e177, 2011.

16. Barker HE, Paget JT, Khan AA and Harrington KJ: The tumour microenvironment after radiotherapy: Mechanisms of resistance and recurrence. Nat Rev Cancer 15: 409-425, 2015.

17. Mizushima N: Autophagy: Process and function. Genes Dev 21: 2861-2873, 2007.

18. Allavena P, Sica A, Solinas G, Porta C and Mantovani A: The inflammatory micro-environment in tumor progression: The role of tumor-associated macrophages. Crit Rev Oncol Hematol 66: 1-9, 2008.

19. Stout RD, Jiang C, Matta B, Tietzel I, Watkins SK and Suttles J: Macrophages sequentially change their functional phenotype in response to changes in microenvironment influences. J Immunol 175: 342-349, 2005.

20. Sica A, Schioppa T, Mantovani A and Allavena P: Tumor-associated macrophages are a distinct M2 polarised population promoting tumor progression: Potential targets of anti-cancer therapy. Eur J Cancer 42: 717-727, 2006.

21. Gocheva V, Wang H-W, Gadea BB, Shree T, Hunter KE, Garfall AL, Berman T and Joyce JA: IL-4 induces cathepsin protease activity in tumor-associated macrophages to promote cancer growth and invasion. Genes Dev 24: 241-255, 2010.

22. Pander J, Heusinkveld M, der Straaten TV, Jordanova ES, Baak-Pablo R, Gelderblom H, Morreau H, van der Burg SH, Guchelaar HJ and van Hall T: Activation of tumor-promoting type 2 macrophages by EGFR-targeting antibody Cetuximab. Clin Cancer Res 17: 5668-5673, 2011.

23. Coffelt SB, Hughes R and Lewis CE: Tumor-associated macrophages: Effectors of angiogenesis and tumor progression. Biochim Biophys Acta 1796: 11-18, 2011.

24. Bingle L, Lewis CE, Corke KP, Reed MW and Brown NJ: Macrophages promote angiogenesis in human breast tumour spheroids in vivo. Br J Cancer 94: 101-107, 2006.

25. Karar J and Maity A: Modulating the tumor microenvironment to increase radiation responsiveness. Cancer Biol Ther 8: 1994-2001, 2009.

26. Amaravadi R, Lippincott Schwartz J, Yin X, Weiss WA, Takebe N, Timmer W, DiPaola RS, Lotze MT and White E: Principles and current strategies for targeting autophagy for cancer treatment. Clin Cancer Res 17: 654-666, 2011.

27. Tan SH, Shui GH, Zhou J, Li JJ, Bay BH, Wenk MR and Shen HM: Induction of autophagy by palmitic acid via protein Kinase C-mediated signaling pathway independent of mTOR. J Biol Chem 287: 14364-14376, 2012.

28. Kanematsu S, Uehara N, Miki H, Yoshizawa K, Kawanaka A, Yuri $\mathrm{T}$ and Tsubura A: Autophagy inhibition enhances sulforaphane-induced apoptosis in human breast cancer cells. Anticancer Res 30: 3381-3390, 2010. 
29. Essafi-Benkhadir K, Refai A, Riahi I, Fattouch S, Karoui H and Essafi M: Quince (Cydonia oblonga Miller) peel polyphenols modulate LPS-induced inflammation in human THP-1-derived macrophages through NF- $\mathrm{B}$, p38MAPK and Akt inhibition. Biochem Biophys Res Commun 418: 180-185, 2012.

30. Spencer M, Yao-Borengasser A, Unal R, Rasouli N, Gurley CM, Zhu B, Peterson CA and Kern PA: Adipose tissue macrophages in insulin-resistant subjects are associated with collagen VI and fibrosis and demonstrate alternative activation. Am J Physiol Endocrinol Metab 299: E1016-E1027, 2010.

31. Qin Z: The use of THP-1 cells as a model for mimicking the function and regulation of monocytes and macrophages in the vasculature atherosclerosis. Proc Natl Acad Sci USA 221: 2-11, 2012.

32. Zheng LD, Xiong ZF, Zhu JW and Wang ZH: Effects of Smac gene over-expression on the radiotherapeutic sensitivities of cervical cancer cellline HeLa. Chin Med J (Engl) 8: 226-30, 2008.

33. Giagkousiklidis S, Vogler M, Westhoff MA, Kasperczyk H, Debati KM and Fulda S: Sensitization for gamma-irradiation-induced apoptosis by second mitochondria-derived activator of caspase. Cancer Res 65: 10502-10513, 2005.
34. Kohli M, Yu J, Seaman C, Bardelli A, Kinzler KW, Vogelstein B, Lengauer $C$ and Zhang L: SMAC/Diablo-dependent apoptosis induced by nonsteroidal antiinflammatory drugs (NSAIDs) in colon cancer cells. Proc Natl Acad Sci USA 101: 16897-16902, 2004.

35. Sreevalsan S, Jutooru I, Chadalapaka G, Walker M and Safe S: 1,1-Bis(3'-indolyl)-1-(p-bromophenyl)methane and related compounds repress survivin and decrease $\gamma$-radiation induced survivin in colon and pancreatic cancer cells. Int J Oncol 35: 1191-1199, 2009.

36. Yu L-W, Ma X-T: Stat3 signaling pathway regulates the expression of Survivin and promotes apoptosis of human colon cancer cells. Zhong Hua Shi Yan Wai Ke Za Zhi She 3: 291-293, 2008 (In Chinese).

37. Rödel C, Haas J, Groth A, Grabenbauer GG, Sauer R and Rödel F: Spontaneous and radiation-induced apoptosis in colorectal carcinoma cells with different intrinsic radiosensitivities: Survivin as a radioresistance factor. Int J Radiat Oncol Biol Phys 55: 1341-1347, 2003.

38. Zhang Y, Teng Z, Yu L and Zhang J: Tumor-associated macrophages affect biological behavior of SW480 cell-line. Acad Med 33: 71-75, 2011. 